\title{
Incompatibility of the Copenhagen interpretation with quantum mechanics formalism
}

\author{
Yuri A. Rylov \\ Institute for Problems in Mechanics, Russian Academy of Sciences, \\ 101-1, Vernadskii Ave., Moscow, 119526, Russia. \\ e-mail: rylov@ipmnet.ru
}

\begin{abstract}
It is proved the mathematical theorem, that the wave function describes the statistical ensemble of particles, but not a single particle. Supposition, that the wave function describes a single particle appears to be incompatible with formalism of quantum mechanics.
\end{abstract}

Interest to interpretation of quantum mechanics was very large from the very beginning of the quantum mechanics creation up to now. Some scientific journals (Physics Today (1999), Uspechi Fizicheskich Nauk (2002)) organized discussions devoted to problems of quantum measurements and their interpretation. There are scientists [1, 2, 3, which believe that the wave function describes a statistical ensemble. There are scientists [4, 5, 6, 7], which believe that the wave function describes a single particle. There are scientists [8, 9, 10], whose position is intermediate. There is a lot of papers devoted to interpretation of quantum mechanics. All discussions were produced on the verbal level. None of researchers had not set the problem mathematically: Which of interpretations does follow from the quantum mechanics formalism? or in negative form: Which of interpretations is incompatible with the quantum mechanics formalism? Such a statement of the problem seems to be very reasonable. However, the question in such a form was not set. After mathematical solution of this problem any discussion on the verbal level seems to be useless.

In this paper we prove a very important theorem, which claims that the wave function may not describe an individual quantum particle. It describes always a statistical ensemble of quantum particles. We shall show, that the action $\mathcal{A}_{\mathrm{S}}$ for the Schrödinger particle $\mathcal{S}_{\mathrm{S}}$ (the dynamic system described by the Schrödinger equation) turns into the action $\mathcal{A}_{\mathcal{E}\left[\mathcal{S}_{\mathrm{cl}}\right]}$ for the statistical ensemble $\mathcal{E}\left[\mathcal{S}_{\mathrm{cl}}\right]$ of free classical particles $\mathcal{S}_{\mathrm{cl}}$, when the quantum constant $\hbar \rightarrow 0$. Such a transition is possible only in the case, when the wave function $\psi$ describes a statistical ensemble of quantum particles, but not a single particle. 
For the free Schrödinger particle $\mathcal{S}_{\mathrm{S}}$ the action has the form

$$
\mathcal{S}_{\mathrm{S}}: \quad \mathcal{A}_{\mathrm{S}}\left[\psi, \psi^{*}\right]=\int\left\{\frac{i \hbar}{2}\left(\psi^{*} \partial_{0} \psi-\partial_{0} \psi^{*} \cdot \psi\right)-\frac{\hbar^{2}}{2 m} \nabla \psi^{*} \nabla \psi\right\} d t d \mathbf{x}
$$

where $\psi=\psi(t, \mathbf{x})$ is a complex one-component wave function, $\psi^{*}=\psi^{*}(t, \mathbf{x})$ is the complex conjugate to $\psi$, and $m$ is the particle mass. It is supposed that in the classical limit $\hbar \rightarrow 0$ the description of the dynamic system $\mathcal{S}_{\mathrm{S}}$ becomes to be a classical description of a free particle $\mathcal{S}_{\mathrm{cl}}$.

However, there are two different classical descriptions of the free classical particle $\mathcal{S}_{\mathrm{cl}}$. The individual classical particle $\mathcal{S}_{\mathrm{cl}}$ is described by the action

$$
\mathcal{A}_{\mathcal{S}_{\mathrm{cl}}}[\mathbf{x}]=\int \frac{m}{2}\left(\frac{d \mathbf{x}}{d t}\right)^{2} d t
$$

where $\mathbf{x}=\left\{x^{1}(t), x^{2}(t), x^{3}(t)\right\}$.

Statistical ensemble $\mathcal{E}\left[\mathcal{S}_{\mathrm{cl}}\right]$ of free classical particles $\mathcal{S}_{\mathrm{cl}}$ is described by the action

$$
\mathcal{A}_{\mathcal{E}\left[\mathcal{S}_{\mathrm{cl}}\right]}[\mathbf{x}]=\int \frac{m}{2}\left(\frac{d \mathbf{x}}{d t}\right)^{2} d t d \boldsymbol{\xi}
$$

where $\mathbf{x}=\left\{x^{1}(t, \boldsymbol{\xi}), x^{2}(t, \boldsymbol{\xi}), x^{3}(t, \boldsymbol{\xi})\right\}$. Parameters $\boldsymbol{\xi}=\left\{\xi_{1}, \xi_{2}, \xi_{3}\right\}$ label elements (particles) of the statistical ensemble $\mathcal{E}\left[\mathcal{S}_{\mathrm{cl}}\right]$. Both dynamic systems $\mathcal{S}_{\mathrm{cl}}$ and $\mathcal{E}\left[\mathcal{S}_{\mathrm{cl}}\right]$ are classical. However, $\mathcal{S}_{\mathrm{cl}}$ has six degrees of freedom (the order of the system of the first order ordinary differential equations), whereas the statistical ensemble $\mathcal{E}\left[\mathcal{S}_{\mathrm{cl}}\right]$ has infinite number of the freedom degrees, because it consists of the infinite number of the particles $\mathcal{S}_{\mathrm{cl}}$. The dynamic system $\mathcal{E}\left[\mathcal{S}_{\mathrm{cl}}\right]$ may be interpreted as an ideal fluid without pressure. This fluid may be described in terms of a wave function [11]. In this case the action (3) has the form

$$
\mathcal{A}\left[\psi, \psi^{*}\right]=\int\left\{\frac{i b}{2}\left(\psi^{*} \partial_{0} \psi-\partial_{0} \psi^{*} \psi\right)-\frac{b^{2}}{2} \boldsymbol{\nabla} \psi^{*} \boldsymbol{\nabla} \psi+\frac{b^{2}}{8 \rho}\left(\rho^{2} \boldsymbol{\nabla} s_{\alpha} \boldsymbol{\nabla} s_{\alpha}+(\boldsymbol{\nabla} \rho)^{2}\right)\right\} d^{4} x
$$

where $b$ is a real constant $b \neq 0, \psi=\left(\begin{array}{l}\psi_{1} \\ \psi_{2}\end{array}\right)$ is a two-component complex wave function and $\psi^{*}=\left(\psi_{1}^{*}, \psi_{2}^{*}\right)$ is the complex conjugate to $\psi$.

$$
\rho=\psi^{*} \psi, \quad s_{\alpha}=\frac{\psi^{*} \sigma_{\alpha} \psi}{\rho}, \quad \alpha=1,2,3
$$

and $\sigma_{\alpha}, \alpha=1,2,3$ are the Pauli matrices. In the case when the flow is irrotational, the wave function $\psi$ may be chosen one-component. In this case $s_{\alpha}=$ const and the action (41) turns into the action

$$
\mathcal{A}\left[\psi, \psi^{*}\right]=\int\left\{\frac{i b}{2}\left(\psi^{*} \partial_{0} \psi-\partial_{0} \psi^{*} \psi\right)-\frac{b^{2}}{2} \nabla \psi^{*} \nabla \psi+\frac{b^{2}}{8 \rho}(\boldsymbol{\nabla} \rho)^{2}\right\} d^{4} x
$$


Let us investigate, into what classical dynamic system $\left(\mathcal{S}_{\mathrm{cl}}\right.$ or $\left.\mathcal{E}\left[\mathcal{S}_{\mathrm{cl}}\right]\right)$ turns the dynamic system $\mathcal{S}_{\mathrm{S}}$ in the limit $\hbar \rightarrow 0$ ?

The quantum constant $\hbar$ is a parameter of the dynamic system (11). As a rule, a change of a parameter of a dynamic system does not change the number and the character of dynamic equations. The number of the freedom degrees does not changes also. The dynamic system $\mathcal{S}_{\mathrm{S}}$ has infinite number of the freedom degrees, and we should expect that at $\hbar \rightarrow 0$ the dynamic system $\mathcal{S}_{\mathrm{S}}$ turns into $\mathcal{E}\left[\mathcal{S}_{\mathrm{cl}}\right]$, which also has infinite number of the freedom degrees, but not into $\mathcal{S}_{\mathrm{cl}}$, which has six degrees of freedom.

However, at $\hbar=0$ the description by means of the action (11) degenerates, and one should consider the limit $\hbar \rightarrow 0$ of the description by means of the action (1). To obtain this limit, we make a change of variables

$$
\psi \rightarrow \Psi_{b}=|\psi| \exp \left(\frac{\hbar}{b} \log \frac{\psi}{|\psi|}\right), \quad \psi=\left|\Psi_{b}\right| \exp \left(\frac{b}{\hbar} \log \frac{\Psi_{b}}{\left|\Psi_{b}\right|}\right)
$$

where $b \neq 0$ is some real constant. After this change of variables the action (11) turns into

$$
\mathcal{A}_{\mathcal{S}_{\mathrm{q}}}\left[\Psi_{b}, \Psi_{b}^{*}\right]=\int\left\{\frac{i b}{2}\left(\Psi_{b}^{*} \partial_{0} \Psi_{b}-\partial_{0} \Psi_{b}^{*} \cdot \Psi_{b}\right)-\frac{b^{2}}{2 m} \nabla \Psi_{b}^{*} \nabla \Psi_{b}-\frac{\hbar^{2}-b^{2}}{2 m}\left(\nabla\left|\Psi_{b}\right|\right)^{2}\right\} d t d \mathbf{x}
$$

The transformation (7) is analytical for any values of parameters $b$ and $\hbar$, except for the case, when $\hbar=0$ or $b=0$. The constant $b$ is arbitrary, and it always can be chosen $b \neq 0$. The value $\hbar=0$ is not considered, because in this case the action (1), as well as the transformation (7) degenerate. For all values of $\hbar \neq 0$ the dynamic systems (11) and (8) are equivalent. At $\hbar \rightarrow 0$ the dynamic system (8) does not degenerate, it turns into the dynamic system $\mathcal{E}^{\prime}\left[\mathcal{S}_{\mathrm{cl}}\right]$

$\mathcal{A}_{\mathcal{E}^{\prime}\left[\mathcal{S}_{\mathrm{cl}}\right]}\left[\Psi_{b}, \Psi_{b}^{*}\right]=\int\left\{\frac{i b}{2}\left(\Psi_{b}^{*} \partial_{0} \Psi_{b}-\partial_{0} \Psi_{b}^{*} \cdot \Psi_{b}\right)-\frac{b^{2}}{2 m} \nabla \Psi_{b}^{*} \nabla \Psi_{b}+\frac{b^{2}}{2 m}\left(\boldsymbol{\nabla}\left|\Psi_{b}\right|\right)^{2}\right\} d t d \mathbf{x}$

which may be considered as the limit of the action (1) at $\hbar \rightarrow 0$. The action (9) is a partial case of the action (3), because (9) coincides with (6), if one takes into account that $\left|\Psi_{b}\right|=|\psi|=\sqrt{\rho}$. Thus, dynamic system $\mathcal{E}^{\prime}\left[\mathcal{S}_{\mathrm{cl}}\right]$ is a special case of the dynamic system $\mathcal{E}\left[\mathcal{S}_{\mathrm{cl}}\right]$

But independently of, whether or not dynamic systems $\mathcal{E}^{\prime}\left[\mathcal{S}_{\mathrm{cl}}\right]$ and $\mathcal{E}\left[\mathcal{S}_{\mathrm{cl}}\right]$ coincide, the dynamic system (9) cannot coincide with the dynamic system (2), because the dynamic system (21) has six degrees of freedom, whereas the dynamic system (9) has infinite number of the freedom degrees. It means that the wave function may not describe a single particle, and the Copenhagen interpretation and other QM interpretations, founded on the statement, that the wave function describes a single particle, may not be used. In particular, such phenomena as superluminal interaction in the EPR experiment and many-worlds interpretation [12, 13] appear to be impossible as founded on the statement, that the wave function describes an individual particle. 


\section{References}

[1] D. I. Blokhintsev, Foundation of Quantum Mechanics. Nauka, 1976. (in Russian). English translation D.I. Blokhintsev, Principles of Quantum Mechanics, allyn and Bacon, Boston, 1964.

[2] L.E. Ballentine, Rev. Mod. Phys., 72, 358. (1970).

[3] L.E. Ballentine, Quantum Mechanics, World Scientific, Singapore, 1998.

[4] W. Heisenberg, in Niels Bohr and the Development of Physics. ed. W.Pauli , London. Pergamon Press Ltd., 1955.

[5] C. Piron, Helv. Phys. Acta 37, 439 (1964)

[6] R. Penrose, The Large, the Small and the Human Mind, CUP (1997)

[7] R.G. Newton, Am. J. Phys. 72(3), 348 (2004).

[8] G. Ludwig, Z. Phys. 181, 233 (1964).

[9] G. Ludwig, Z. Naturforsch. 22a, 1303 (1967).

[10] G. Ludwig, Commun. Math. Phys. 37, 221 (1974).

[11] Yu. A. Rylov, J. Math. Phys. 40, 256, (1999).

[12] H. Everett, Rev. Mod. Phys. 29454 (1957); Reprinted in Quantum Theory and Measurement (Eds. J.A. Wheeler, W.H. Zurek) (Princeton, N.J.: Princeton University Press. 1983)

[13] B.S. DeWitt and N. Graham (Eds) The Many-Worlds Interpretation of Quantum Mechanics (Princeton, N.J.: Princeton University Press, 1973) 